\title{
Silica nanoparticles induce reversible damage of spermatogenic cells via RIPKI signal pathways in $\mathrm{C} 57$ mice
}

This article was published in the following Dove Press journal:

International Journal of Nanomedicine

24 May 2016

Number of times this article has been viewed

\author{
Lihua Ren ${ }^{1,2}$ \\ Jin Zhang ${ }^{1,2}$ \\ Yang Zou ${ }^{1,2}$ \\ Lianshuang Zhang ${ }^{1,2}$ \\ Jialiu Wei ${ }^{1,2}$ \\ Zhixiong Shi, ${ }^{1,2}$ \\ Yanbo $\mathrm{Li}^{1,2}$ \\ Caixia Guo ${ }^{2}$ \\ Zhiwei Sun ${ }^{1,2}$ \\ Xianqing Zhou ${ }^{1,2}$ \\ 'Department of Toxicology and \\ Hygienic Chemistry, School of Public \\ Health, ${ }^{2}$ Beijing Key Laboratory of \\ Environmental Toxicology, Capital \\ Medical University, Beijing, People's \\ Republic of China
}

Correspondence: Xianqing Zhou Department of Toxicology and Hygienic Chemistry, School of Public Health, Capital Medical University, Nol0 Xi Tou Tiao, You An Men, Beijing, 100069, People's Republic of China

Tel +8610 839। 1775

Fax +86 I0 5978 47। 9

Email xqzhou@ccmu.edu.cn
Abstract: The reproductive toxicity of silica nanoparticles (SiNPs) is well known, but the underlying mechanism is still not clear. To investigate the toxic mechanism of SiNPs on spermatogenic cells, 60 C57 male mice were randomly and equally divided into three groups (the control group, the saline control group, and the SiNPs group) with two observed time points (45 days and 75 days). The mice in the SiNPs group were administered with SiNPs $2 \mathrm{mg} / \mathrm{kg}$ diluted in normal saline, and the mice of the saline control group were given equivoluminal normal saline by tracheal perfusion every 3 days for 45 days (in total 15 times). The control group mice were bred without treatment. In each group, a half number of the mice were sacrificed on the 45th day after the first dose, and the remaining half were sacrificed on the 75th day. The results showed that SiNPs increased the malformation of sperms and decreased the motility and concentration of sperms in epididymis on the 45th day after the first dose. SiNPs induced oxidative stress in testis and led to apoptosis and necroptosis of the spermatogenic cells. Furthermore, SiNPs increased the expression of Fas/FasL/RIPK1/FADD/caspase-8/caspase-3 and RIPK3/MLKL on the 45th day after the first dose. However, compared with the saline control group, the index of sperms and the expression of Fas/FasL/RIPK1/FADD/caspase-8/ caspase-3/RIPK3/MLKL showed no significant changes in the SiNPs group on the 75th day after the first dose. These data suggested that SiNPs could induce apoptosis and necroptosis in the spermatogenic cells by activating the RIPK1 pathway resulting from oxidative stress in male mice. SiNPs-induced damage recovered on the 75th day after the first dose, which suggested that SiNPs-induced toxicity is reversible.

Keywords: silica nanoparticles, RIPK1 signal pathway, apoptosis, necroptosis, spermatogenic cells, reversible

\section{Introduction}

With the development of engineered nanoparticles (ENPs), numerous ENPs like metal nanoparticles (NPs), magnetic NPs, quantum dots, and silica NPs (SiNPs) have been widely used in the delivery of drugs, therapeutics, diagnostics, vaccines, and nucleotides. ${ }^{1,2}$ SiNPs are widespread nanomaterials used in toothpastes, foods, for delivery of drugs, and cancer detection. ${ }^{3-5}$ In addition, in the nature, SiNPs mainly exist in soil, sand, ore, and particulate matter. ${ }^{6,7}$ Due to increasing atmospheric pollution, enhanced concentrations of SiNPs are found in the environment. ${ }^{2,8}$ There is a concern that research on the possible health risks of SiNPs are not keeping pace with the rapid growth in the number of SiNP products.

Previous researches have shown that many kinds of NPs could impair male reproductive system and that SiNPs could cause lesions in Sertoli cells and spermatogenic 
cells. $^{9-11}$ Thus, SiNPs could cause damage to sperms, leading to sperm malformation and decrease in the number and motility of sperms. ${ }^{12,13}$ Our previous study showed that SiNPs caused damage to mitochondrial cristae which, in turn, led to a decrease in the quantity and quality of epididymal sperms. But SiNPs-induced damage recovered after stopping the exposure. ${ }^{14}$ However, controversial reports also exist. The previous study showed that short-term exposure to NPs could not induce the damage to reproduction. ${ }^{15}$ Therefore, there is an urgent need to investigate the toxicological mechanism of SiNPs on reproduction.

Oxidative stress has been considered as an initiating event of reproductive toxicity. The studies also showed that exposure to SiNPs could induce oxidative stress, inflammation, apoptosis, and organ injury. ${ }^{16-18}$ Our previous study showed that exposure to SiNPs could damage the mitochondria and lead to energy metabolism dysfunction. ${ }^{14}$ However, the molecular mechanisms involved in the cytotoxicity of SiNPs on spermatogenic cells remain unclear and poorly understood. In this study, we examined the RIPK1 signaling pathway to explore the possible mechanism of the cytotoxicity induced by SiNPs. These findings could provide persuasive evidence for the cytotoxicity effects of SiNPs on reproduction.

\section{Materials and methods Experimental design}

Sixty C57 clean grade male mice were purchased from the Beijing Vital River Laboratory Animal Technology Co., Ltd. (Animal production license number: SCXK2012-0001) (Beijing, People's Republic of China). The mice were aged 6-7 weeks with a mean weight of $20.1 \pm 2.0 \mathrm{~g}$. Every five mice were raised in a plastic cage $(26 \mathrm{~cm} \times 15 \mathrm{~cm} \times 15 \mathrm{~cm})$ in a ventilated animal care facility. The temperature of the house was maintained at $22^{\circ} \mathrm{C} \pm 2^{\circ} \mathrm{C}$, and the relative humidity was kept at $60 \% \pm 10 \%$. The animals were raised 12 hour: 12 hour light/dark cycle. All the mice were provided food and drinking water ad libitum. The pad was changed every 3 days. Animal protocols were approved by the Capital Medical University Institutional Animal Care and Use Committee (Ethical review number: AEEI-2014-068) and conformed to the US National Institutes of Health Guide for the Care and Use of Laboratory Animals. Prior to experiment, the mice were adapted to the environment for 1 week. Then, 60 healthy adult male mice were randomly and equally divided into three groups: the control group, the saline control group, and the SiNPs group. The mice in the SiNPs group were administered with SiNPs $2 \mathrm{mg} / \mathrm{kg}$ diluted in normal saline, with diameters of $57.66 \mathrm{~nm}$, and the mice in the saline control group were given equivoluminal normal saline by tracheal perfusion every 3 days for 45 days (in total 15 times). The control group mice were bred without treatment. The mice were injected intraperitoneally with $7 \mu \mathrm{L} / \mathrm{g}$ body weight of 5\% chloral hydrate anesthesia before performing the experiment after 12 hours of fasting. A half of the mice in every group were sacrificed at 45 days, and the remaining half were sacrificed at 75 days after the first dose. Testes and epididymides were collected from each animal for analysis. The sperms in the epididymides were collected immediately, and the testes were weighed; then, the right testes were fixed, and the left testes were stored at $-80^{\circ} \mathrm{C}$ until analysis.

\section{SiNPs preparation and characterization}

SiNPs were prepared and characterized as reported in our previous laboratory study. ${ }^{19}$ Briefly, SiNPs were produced in the College of Chemistry, Jilin University, People's Republic of China, by the Stöber method. Ethanol solution $(50 \mathrm{~mL})$, ammonia $(2 \mathrm{~mL})$, and water $(1 \mathrm{~mL})$ were mixed, and then tetraethyl orthosilicate $(2.5 \mathrm{~mL})$ was added to the mixture. The mixture was kept stirring $(150 \mathrm{rpm})$ at $40^{\circ} \mathrm{C}$ for 12 hours, and then the particles were obtained by centrifugation (12,000 rpm) for 15 minutes, washed three times, and dispersed in deionized water $(50 \mathrm{~mL})$. To avoid the aggregation of SiNPs, biodegradable and low critical micelle concentration surfactants were used according to the procedure described in the previous study. ${ }^{20}$ Finally, the surfactants were washed off, and the suspensions of SiNPs were sonicated by a sonicator (160 W, 20 kHz, 5 minutes) (Bioruptor UDC-200; Diagenode, Liège, Belgium) before addition to dispersion solution and performing tracheal perfusion in order to minimize their possible aggregation. The particles were observed by transmission electron microscopy (JEM-2100; JEOL, Tokyo, Japan). The diameter of SiNPs was measured by computer-assisted image analysis software (ImageJ software; National Institutes of Health, Bethesda, MD, USA). The zeta potential and hydrodynamic sizes of SiNPs were determined with a Zetasizer (Nano ZS90; Malvern Instruments, Malvern, UK).

\section{Evaluation of sperms in epididymides}

Sperms were collected from epididymides and placed in Dulbecco's Modified Eagle Medium (2 mL) at $37^{\circ} \mathrm{C}$ for 5 minutes, and then the concentration and the motility of sperms were analyzed by computer-assisted semen analyzer (Hamilton Thorne IVOS-II; Hamilton Thorne Research, Beverly, MA, USA). The results regarding the concentration and the motility of sperms were displayed on the computer. A smear of the sperm suspension was made on the glass 
slides and was used for assessing the malformation of sperms after hematoxylin-eosin staining. A total of 1,000 sperms on every glass slide were counted to determine the proportion of malformed sperms using an optical microscope (Olympus BX53; Olympus Corporation, Tokyo, Japan).

The sperm $=\frac{\text { The malformed sperm number }}{1,000} \times 100 \%$

\section{Evaluation of histological structure and ultrastructure of testes}

The evaluation of histological and ultrastructure of testes was performed using standard laboratory procedures. Briefly, the collected testes were rapidly fixed in picric acid, dehydrated, embedded in paraffin blocks, and placed on separate glass slides. The stained sections were examined by a histopathologist using an optical microscope (Olympus BX53). One testicle was randomly removed immediately after the mice were sacrificed for observation by electron microscopy. The testicle was first injected with $2.5 \%$ glutaraldehyde and kept for 30 minutes. Then it was cut into small pieces and placed in the $2.5 \%$ glutaraldehyde solution at $4^{\circ} \mathrm{C}$ for 24 hours. The pieces were washed three times for 10 minutes each with phosphate buffer at pH 7.2 and then dehydrated with ethanol. Subsequently, the dehydrated samples were embedded with Epon 812, sliced by an LKB-V microtome, and stained with $3 \%$ uranyl acetate-lead citrate. Finally, we used JEM-1400 and JEM-2100 transmission electron microscopes (JEOL) to observe the testicular ultrastructures.

\section{Determination of the apoptosis of spermatogenic cells in the testes}

One-step terminal deoxynucleotidyl transferase (TdT)mediated dUTP nick-end labeling (TUNEL) apoptosis assay kit (KeyGen Biotechnology, Nanjing, People's Republic of China) was used to confirm the presence of apoptotic cells in the paraffin-embedded sections, which were dewaxed by treating with dimethyl benzene for two times, immersed in gradient alcohol, and then in distilled water. TUNEL assay was performed according to the manufacturer's instructions. TUNEL-positive cells were observed by a fluorescence microscope (Nikon Eclipse Ti-U; Nikon Corporation, Tokyo, Japan) after green fluorescent staining. To quantify the expression of apoptosis cells, the intensity of green fluorescence was measured using computer-assisted image analysis (LAS Image Analysis V4.0; Leica Microsystems, Wetzlar, Germany).

\section{Determination of oxidative stress indices in the testes}

The total protein of the testes tissue was extracted according to the instructions of a protein extraction kit (Jiancheng Bioengineering Institute, Nanjing, People's Republic of China). The protein concentration of testes was assayed using a protein quantification kit (KeyGen Biotechnology). The levels of superoxide dismutase (SOD) and malondialdehyde (MDA) were measured using commercial kits (Jiancheng Bioengineering Institute) according to the manufacturer's protocols. The concentration of 3-nitrotyrosine (3-NT) was assayed using a 3-NT ELISA Kit (Shanghai Yuping Biotechnology Limited Company, Yuping, People's Republic of China) according to the manufacturer's instructions.

\section{Determination of protein expression in RIPKI signal pathway}

In order to determine the expression of proteins in RIPK1 signal pathway, Western blots were performed. The total protein of the testes tissue was extracted using a protein extraction kit (Jiancheng Bioengineering Institute) and measured by the protein quantification kit (KeyGen Biotechnology). Extracts containing equal amounts of $40 \mu \mathrm{g}$ of lysate proteins were used. The protein samples were subjected to sodium dodecyl sulfate-polyacrylamide gel electrophoresis in 15\% separating gels and 5\% stacking gels and electrophoretically transferred to polyvinylidene fluoride (PVDF) membranes (EMD Millipore, Billerica, MA, USA). After blocking with 5\% bovine serum albumin (KeyGen Biotechnology, Nanjing, People's Republic of China) in Tris-buffered saline-Tween (TBST) for 2 hours at room temperature $\left(20^{\circ} \mathrm{C}-30^{\circ} \mathrm{C}\right)$, the PVDF membrane was incubated with $\beta$-actin/Fas/FasL/RIPK1/ caspase-8/cleaved caspase-8/caspase-3/cleaved caspase-3/ RIPK3/FLIP (1:1,000, rabbit antibodies, Cell Signaling Technology, Beverly, MA, USA), MLKL (1:500, goat antibodies, Santa Cruz Biotechnology Inc., Dallas, TX, USA), FADD (1:500, rabbit antibodies, Santa Cruz Biotechnology Inc.) at room temperature $\left(20^{\circ} \mathrm{C}-30^{\circ} \mathrm{C}\right)$ for 1 hour on a shaking table and at $4^{\circ} \mathrm{C}$ for overnight. After the PVDF membranes were washed three times with TBST every 10 minutes, the PVDF membranes were incubated with anti-rabbit IgG secondary antibody or anti-goat IgG secondary antibody (1:5,000, ZSGB-BIO, Beijing, People's Republic of China) for 1 hour at room temperature $\left(20^{\circ} \mathrm{C}-30^{\circ} \mathrm{C}\right)$ on a shaking table. After the PVDF membranes were washed three times with TBST every 10 minutes, the antibody-bound proteins were detected by the enhanced chemiluminescence reagent (Thermo Fisher Scientific, Waltham, MA, USA) with Chemiluminescence 
imaging system (Tanon 5200; Tanon, Guangzhou, People's Republic of China).

\section{Statistical analyses}

All the statistical analyses were performed by using the statistical software SPSS17.0 (SPSS Inc., Chicago, IL, USA). The independent sample $t$-test was used to test mean differences between the control group and the saline control group and the saline control group and the SiNPs group. All of the values were expressed as the mean value \pm standard error. $P<0.05$ was considered to indicate statistical significance.

\section{Results}

\section{Characterization of SiNPs}

The characterization of SiNPs was done in our previous study. ${ }^{19}$ The shape of SiNPs was found to be near-spherical, and their average diameter was $57.66 \mathrm{~nm}$. The hydrodynamic sizes and zeta potentials of SiNPs detected in the distilled water or Dulbecco's Modified Eagle Medium displayed good monodispersity and excellent stability, and these findings were similar to those reported by Guo et al ${ }^{19}$ (Figure 1).

\section{Effects of SiNPs on murine testes}

On the 45th day after the first dose, the shape of seminiferous tubules and the cell arrangement between the control group and the saline control group were regular, and the cell layer was thick (Figure 2A, B, D, and E). The cellular and

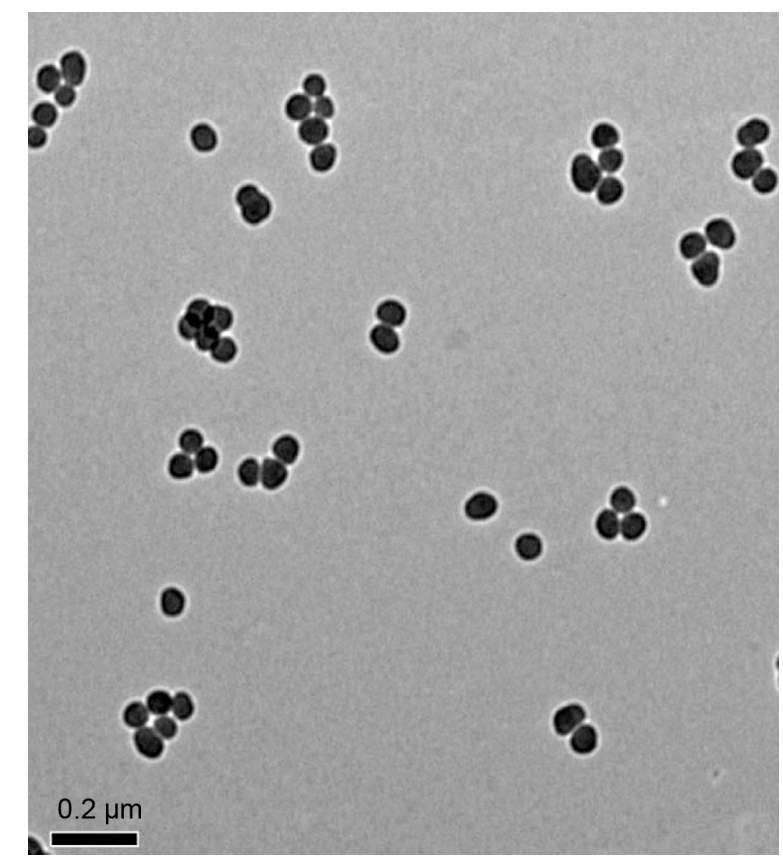

Figure I The characterization of SiNPs.

Notes: TEM image shows SiNPs have a near-spherical shape and good monodispersity, with an average diameter of $57.66 \mathrm{~nm}$.

Abbreviations: SiNPs, silica nanoparticles; TEM, transmission electron microscopy. nuclear membranes were intact (Figure 3A, B, E, and F), and the outer membrane of the mitochondria and the sperm membranes were smooth and intact (Figure 3C, D, G, and H). The data suggested that the experimental operation of tracheal perfusion had no influence on the testes in mice. The rest of the indices manifested no significant difference between the control group and the saline control group, which further indicated that the experimental operation of tracheal perfusion had no effect on the murine reproductive system.

In the SiNPs group, the shape of the seminiferous tubules and the cell arrangement were irregular, cell layer was thin, vacuolization was observed in some seminiferous tubules, exfoliated cells emerged in some seminiferous tubules (Figure 2C and F), and swollen mitochondria were observed (Figure $3 \mathrm{~K}$ ). The cross sections of the outer membrane of the sperm tail were irregular (Figure 3L). Additionally, we also clearly observed SiNPs in the spermatogenic cells of the testis (Figure 3I and J). However, on the 75th day after the first dose, the structure of seminiferous tubules recovered, the vacuolization and exfoliated cells in the tubules disappeared (Figure 2I and L), and the cross sections of the outer membrane of the sperm tail gradually became regular (Figure 3X).

\section{Effects of SiNPs on sperms in epididymides}

On the 45th day after the first dose, the sperm concentration and the sperm motility in the SiNPs group decreased compared with the saline control group. Meanwhile, the sperm abnormity in the SiNPs group was significantly higher than that in the saline control group. On the 75 th day after the first dose, the sperm concentration, the sperm motility, and the sperm abnormity in the SiNPs group had no significant difference compared with the saline control group (Figure 4).

\section{Effects of SiNPs on cell apoptosis in murine testes}

On the 45 th day after the first dose, the cells in the seminiferous tubules in the SiNPs group underwent more apoptosis than the cells in the saline control group. The apoptotic cells were mainly spermatocytes and spermatoblasts. On the 75th day after the first dose, the apoptosis in the SiNPs group and the saline control group displayed no difference (Figure 5).

\section{Effects of SiNPs on the oxidative stress in murine testes}

On the 45th day after the first dose, SOD activity in the SiNPs group was significantly lower than in the saline control 

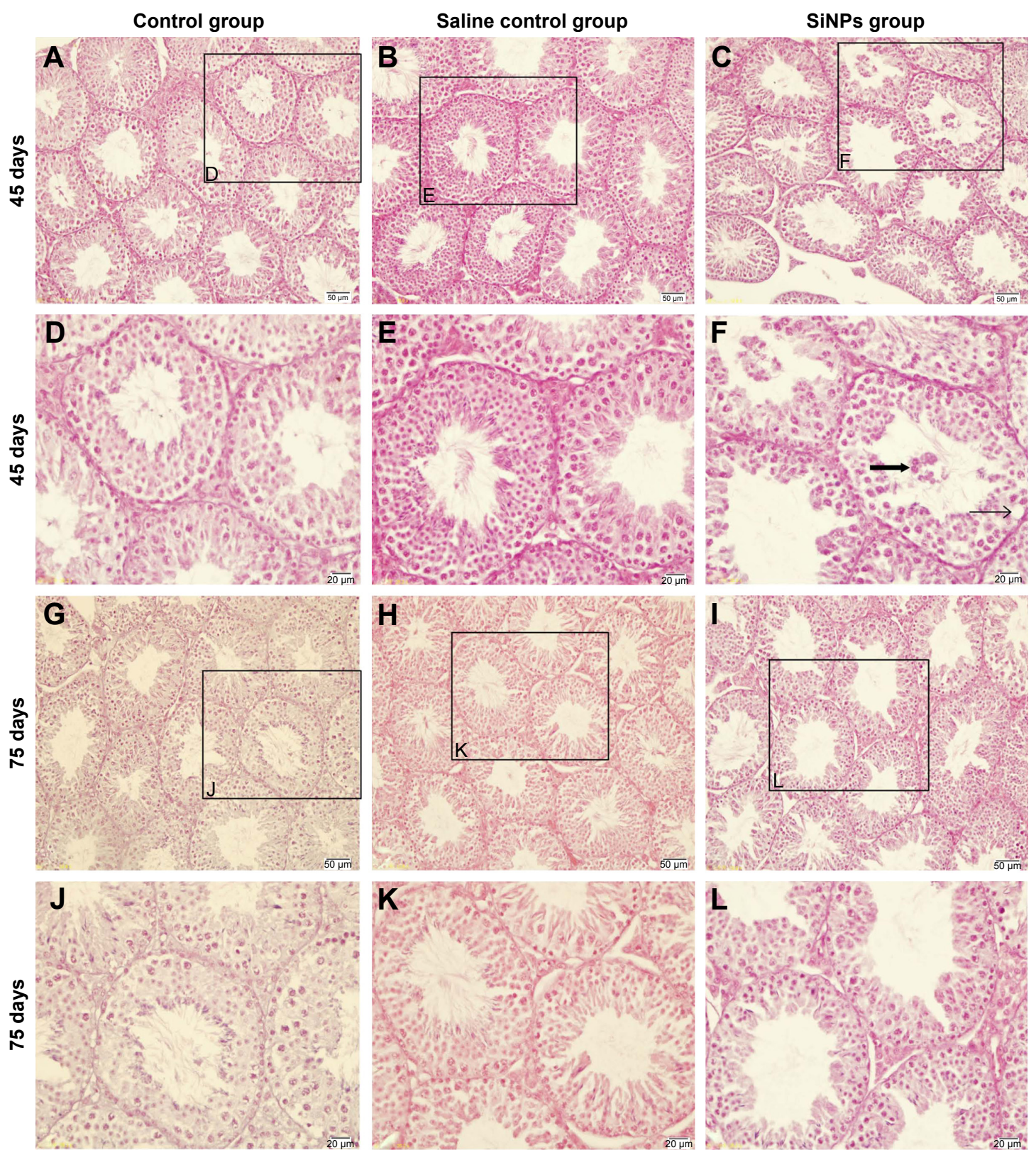

Figure 2 The effects of SiNPs on the structure of testicular tissue in mice.

Notes: (A and D) The shape of seminiferous tubles and the cell arrangement were regular in the control group on the 45 th day after the first dose. (B and $\mathbf{E})$ The shape of seminiferous tubles and the cell arrangement were regular in the saline control group on the 45th day after the first dose. (C and $\mathbf{F}$ ) The shape of seminiferous tubles and the cell arrangement were irregular after exposure to SiNPs for 45 days. SiNPs led to vacuolization and exfoliated cells in some seminiferous tubles. (G and J) The shape of seminiferous tubles and the cell arrangement were regular in the control group on the 75th day after the first dose. $(\mathbf{H}$ and $\mathbf{K})$ The shape of seminiferous tubles and the cell arrangement were regular in the saline control group on the 75th day after the first dose. (I and $\mathbf{L})$ The shape of seminiferous tubles and the cell arrangement were regular in the SiNPs group on the 75th day after the first dose, and the vacuolization and exfoliated cells in some seminiferous tubles induced by SiNPs weren't observed. The figure shows optical microscope images of testis. The thin black arrow represents the vacuolization, and the wide black arrow represents the exfoliated cells. The data indicated that SiNPs could damage the seminiferous epithelium in testis of mice.

Abbreviation: SiNPs, silica nanoparticles.

group. The levels of MDA and 3-NT in the SiNPs group were significantly higher than in the saline control group. On the 75th day after the first dose, SOD activity and 3-NT level showed no significant difference between the SiNPs group and the saline control group. The level of MDA in the SiNP group was higher than in the saline control group $(P=0.048)$ (Figure 6).

\section{Effects of SiNPs on the expression of proteins of RIPKI signal pathway}

Western blot was used to detect the expression of proteins of RIPK1 signal pathway. On the 45th day after the first dose, the expression of death receptor Fas and its ligand FasL was upregulated. Compared with the saline control group, the expression of RIPK1/FADD/caspase-8/cleaved 

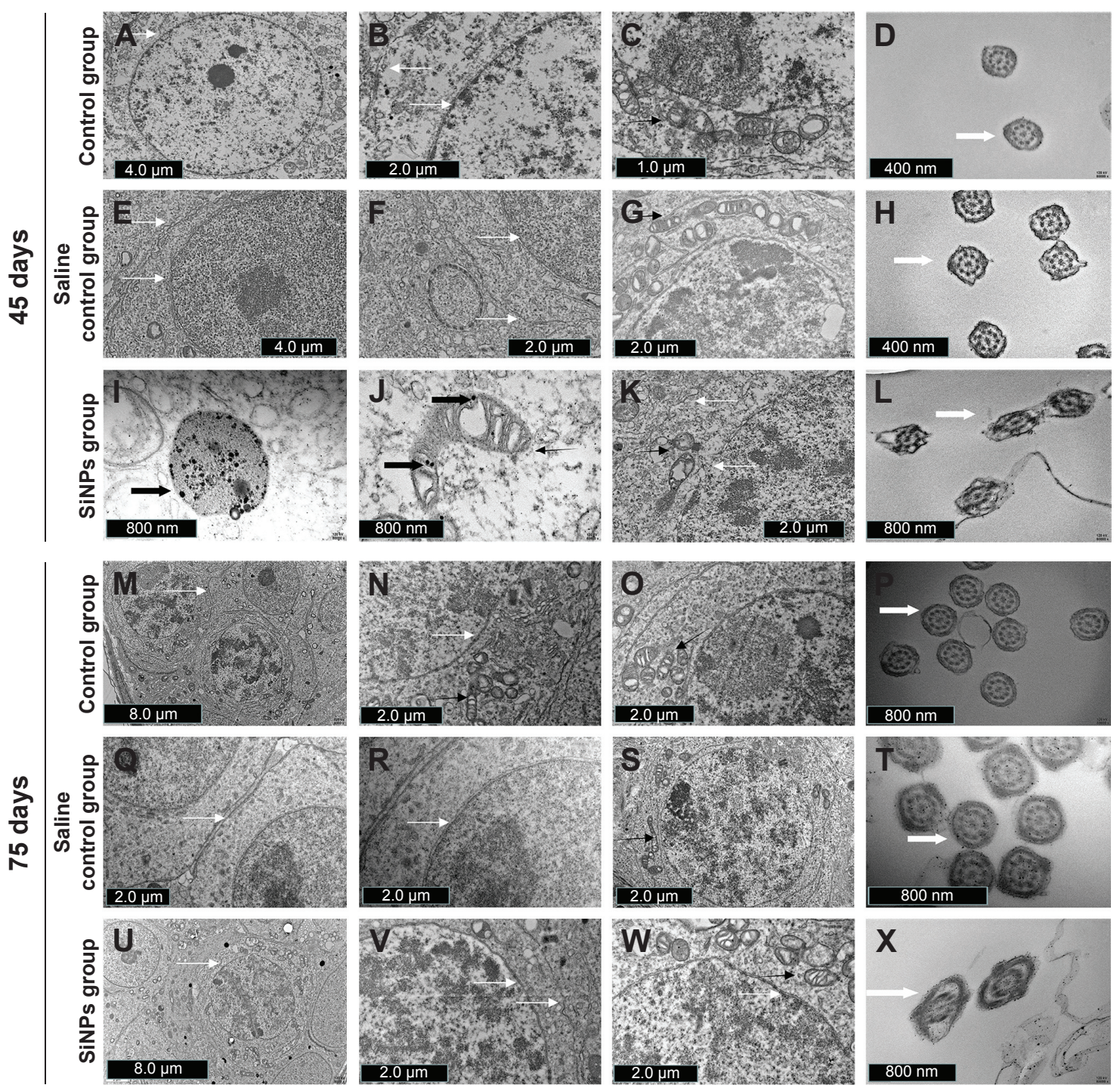

Figure 3 The effects of SiNPs on the ultrastructure of testicular tissue in mice.

Notes: (A-D) The ultrastructure of testes in the control group on the 45th day after the first dose. (E-H) The ultrastructure of testes in the saline control group on the 45th day after the first dose. (I and J) SiNPs were observed in the SiNPs group on the 45th day after the first dose. (K and $\mathbf{L})$ SiNPs led to swollen mitochondria and changed the shape of the cross-sections of the sperm tail in the SiNPs group on the 45th day after the first dose. (M-P) The ultrastructure of testes in the control group on the 75th day after the first dose. (Q-T) The ultrastructure of testes in the saline control group on the 75th day after the first dose. (U-X) The ultrastructure of testes in the SiNPs group on the 75th day after the first dose. The swollen mitochondria weren't observed, and the shape of the cross-sections of the sperm tail were similar to that in the saline control group. The figure shows TEM images of testis. The thin black arrow represents the mitochondria, thin white arrow represents the cell membrane or nuclear membrane, wide white arrow represents the cross sections of the sperms, and wide black arrow represents the SiNPs. The data indicated that SiNPs could accumulate in the testis and damage the mitochondria and sperms.

Abbreviations: SiNPs, silica nanoparticles; TEM, transmission electron microscopy.

caspase-8/caspase-3/cleaved caspase-3 in the apoptosis signal pathway and the expression of RIPK3/MLKL in the necroptosis signal pathway were both enhanced in the SiNPs group. Meanwhile, the expression of survival-promoting molecule FLIP increased in the SiNPs group. Collectively, the results suggested that SiNPs could induce apoptosis and necroptosis via activating RIPK1 signal pathway (Figure 7). On the 75th day after the first dose, the expression of death receptor Fas and its ligand FasL as well as RIPK1/FADD/caspase-8/cleaved caspase-8/caspase-3/cleaved caspase-3/RIPK3/MLKL/FLIP showed no significant difference between the SiNPs group and the saline control group (Figure 8).

\section{Discussion}

Atmospheric exposure is one of the most common ways for human exposure to SiNPs. ${ }^{4,8}$ SiNPs can migrate from lung to extrapulmonary tissues or organs. ${ }^{21,22}$ In our present research, the mice were exposed to SiNPs by endotracheal instillation, as this method simulates the way of the human exposure to SiNPs. After 45 days exposure, SiNPs were 

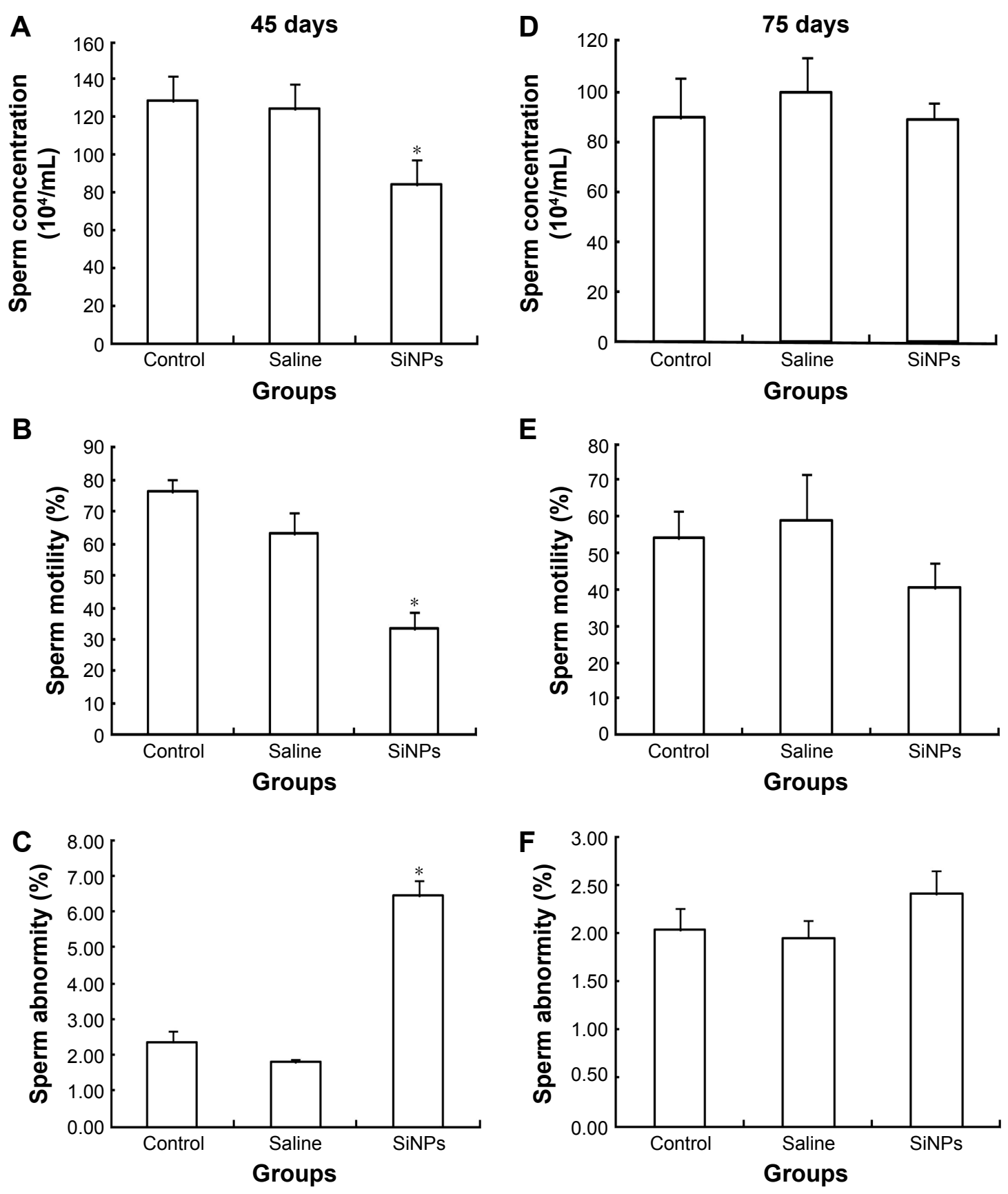

Figure 4 The effects of SiNPs on the quality and quantity of sperms in epididymides.

Notes: (A-C) SiNPs decreased the sperm concentration and the sperm motility, and increased the sperm abnormity on the 45th day after the first dose. (D-F) The sperm concentration, the sperm motility, and the sperm abnormity in the SiNPs group had no significant difference compared with the saline control group on the 75th day after the first dose. Data are expressed as the mean \pm standard error from three independent experiments. $* P<0.05$ vs the saline control group. Saline group represents the saline control group. The data indicated that SiNPs could decrease the quality and quantity of sperms.

Abbreviation: SiNPs, silica nanoparticles.

observed in the testes by transmission electron microscopy, which demonstrated that SiNPs were able to pass the bloodtestis barrier and enter into the testis. This is consistent with another research which showed that NPs could pass blood-testis barrier and accumulate in the testis after exposure to NPs by intravenous infusion. ${ }^{23}$ In addition, SiNPs can also decrease sperm numbers and sperm motility of the male mice. ${ }^{12,13}$ The present study also corroborated that the sperm concentration, the sperm motility, and the sperm abnormity decreased, while the sperm abnormity increased in the SiNPs group compared with the saline control group on the 45th day after the first dose. Our results substantiated that exposure to SiNPs via the respiratory system could lead to lesions in testes and sperms. It is reported that SiNPs can 

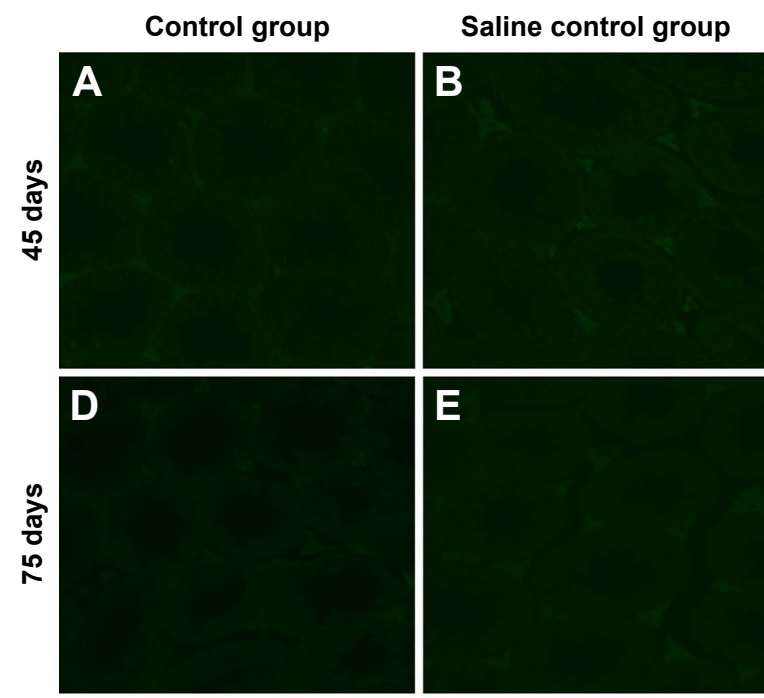

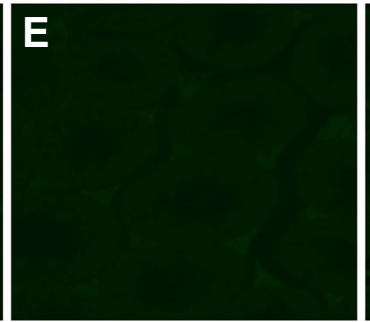

SiNPs group
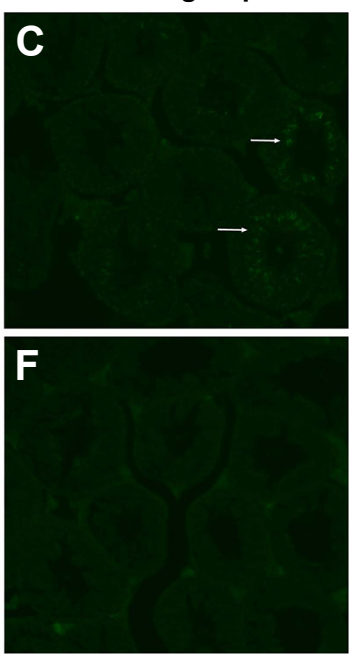
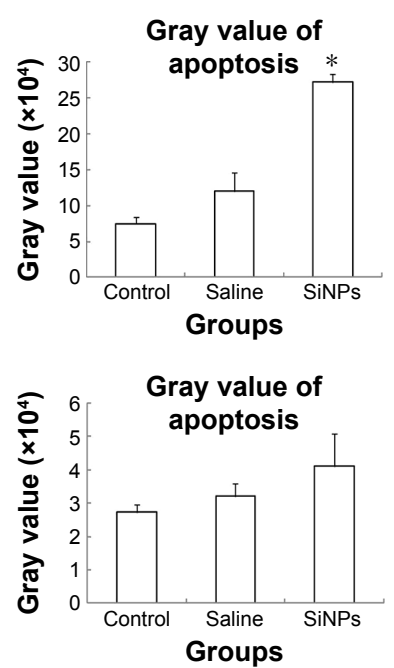

Figure 5 The effects of SiNPs on the apoptosis of spermatogenic cells in testis of mice.

Notes: (A-C) The apoptotic cells were confirmed by TUNEL in every group on the 45th day after the first dose. The apoptotic cells were observed by a fluorescence microscope. SiNPs increased the proportion of apoptosis cells. (D-F) The apoptotic cells were confirmed by TUNEL in every group on the 75th day after the first dose. The apoptosis in the SiNPs group and the saline control group displayed no significant difference on the 75th day after the first dose. The apoptosis of spermatogenic cells was detected by TUNEL assay. Data are expressed as the mean \pm standard error from three independent experiments. $* P<0.05$ vs the saline control group. The white arrow represents the apoptosis of cells. Saline group represents the saline control group. The data indicated that SiNPs could increase the apoptosis of spermatogenic cells. Abbreviations: SiNPs, silica nanoparticles; TUNEL, terminal deoxynucleotidyl transferase (TdT)-mediated dUTP nick-end labeling.

penetrate cytomembrane and sediment in mitochondria or even diffuse into the nucleus and then cause cell death when the diameter of SiNPs is smaller than the size of cellular organelles. ${ }^{24-27}$ The present study indicated that SiNPs caused spermatogenic cells apoptosis, as detected by TUNEL, but the mechanism underlying apoptosis remains unclear.

The study reported that NPs could induce oxidative stress and the testis dysfunction. ${ }^{10}$ The present research showed that SiNPs significantly impaired SOD activity and increased the MDA and 3-NT levels on the 45th day after the first dose. The levels of MDA, SOD, and 3-NT indicate the generation of reactive oxygen species/reactive nitrogen species. ${ }^{28,29}$ Oxidative stress triggered by SiNPs can be one of the crucial mechanisms for exerting their nanotoxicity. ${ }^{14,19,30}$ Oxidative stress might be related to the chemical and surface characteristics of the NPs, ${ }^{31}$ which may be connected with the surface chemical architecture, hydroxyl groups, and compartmentalized cellular modification. ${ }^{32-34}$ Owing to the special characteristics, NPs could interact with the mitochondria after NPs exposure. ${ }^{35}$ Furthermore, NPs can disrupt the electron transport chain, decrease the mitochondrial membrane potential, hinder the $\mathrm{Ca}^{2+}$ uptake, and increase the permeability of the mitochondria, which then leads to mitochondrial damage and finally induces oxidative stress. ${ }^{36,37}$ The generation of oxidative stress implicates imbalance between oxidative and antioxidative systems. Oxidative stress can induce tissue damage in the context of various diseases and lead to cell death through activating regulating pathways of apoptosis or necroptosis. ${ }^{38}$ Oxidative stress has been implicated in mediating apoptosis or necrosis through death receptor pathway. ${ }^{39-41}$ Fas/FasL is a membrane translocator that is composed of intracellular region, transmembrane domain, and extracellular region. Fas/FasL can be stimulated by extrinsic apoptosis pathway. ${ }^{42,43}$ The death domain of Fas/FasL exists in the intracellular region, which interacts with RIPK1 and FADD after FasL binds to Fas.

To obtain further insight into the mechanism of SiNPs-induced apoptosis of spermatogenic cells in testis, we examined the expression of RIPK1/FADD/caspase-8/ caspase- 3 and RIPK3/MLKL by Western blots. RIPK1 is a hub that regulates caspase8-dependent apoptosis, RIPK3/ MLKL-dependent necroptosis, and NF-kB-mediated cell survival. ${ }^{44}$ RIPK1 can be triggered by death receptors. ${ }^{48,49}$ The present research showed that apoptosis rate in the SiNPs groups was higher than in the saline control group on the 45th day after the first dose. Furthermore, we found that SiNPs could increase the expression of RIPK1/FADD/ caspase-8/caspase-3, and RIPK3/MLKL, which suggested that SiNPs induced RIPK1/FADD/caspase-8/caspase-3-dependent apoptosis and RIPK3/MLKL-dependent necroptosis. Meanwhile, the expression of FLIP was increased. FLIP can promote cell survival by competitively binding to caspase- 8 , which in turn impedes the activation 


\section{A}

45 days

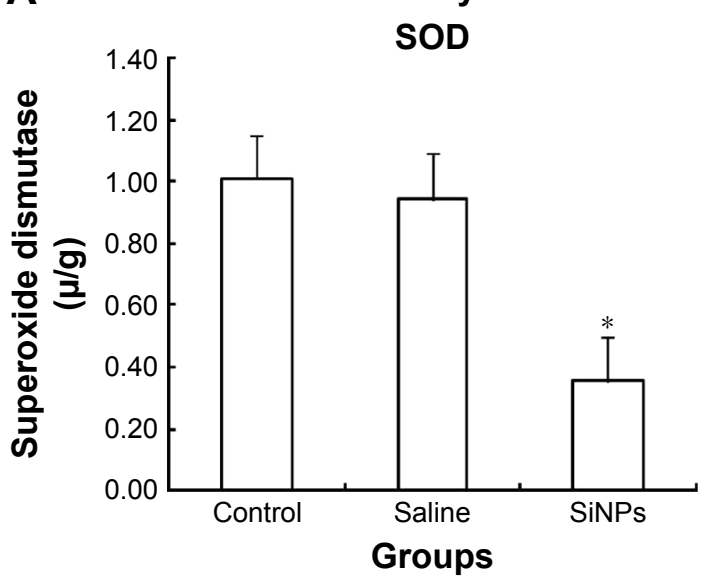

C
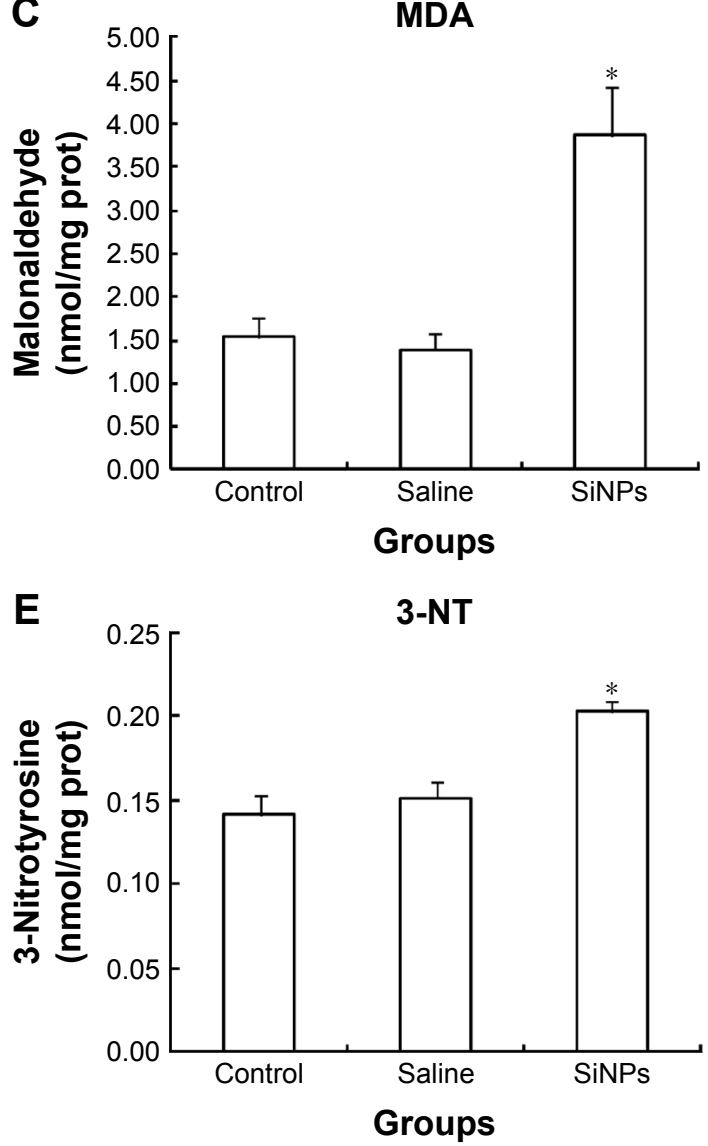
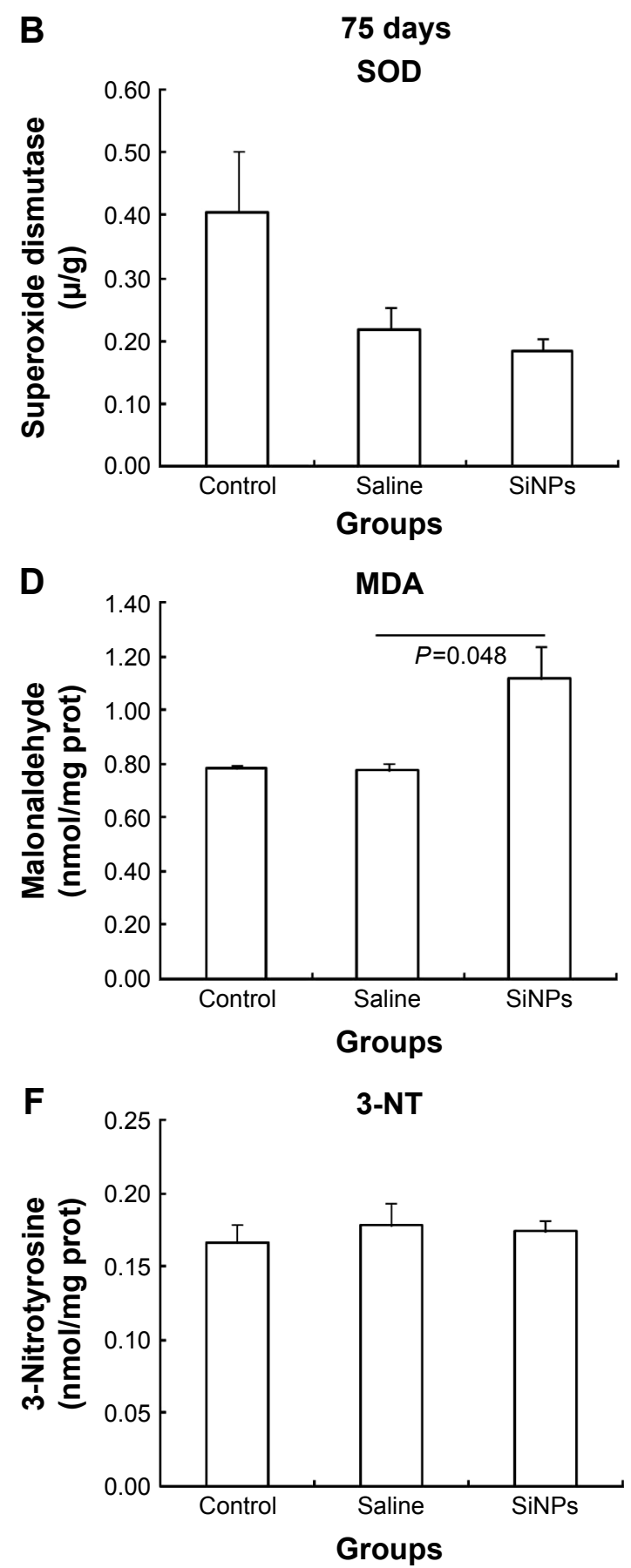

Figure 6 The effects of SiNPs on the oxidative stress in testis of mice.

Notes: (A, C, and E) SiNPs increased the level of SOD, MDA and 3-NT on the 45th day after the first dose. (B, D, and F) The level of SOD and 3-NT showed no significant difference between the SiNPs group and the saline control group on the 75th day after the first dose. The level of MDA in the SiNP group was higher than that in the saline control group on the 75 th day after the first dose $(P=0.048)$. Data are expressed as the mean \pm standard error from three independent experiments. $* P<0.05$ vs the saline control group. Saline group represents the saline control group. The data indicated that SiNPs could increase the level of oxidative stress.

Abbreviations: SOD, superoxide dismutase; MDA, malondialdehyde; 3-NT, 3-nitrotyrosine; SiNPs, silica nanoparticles; prot, protein.

of FADD by caspase- $8 .{ }^{50}$ The increased levels of FLIP can repress cell apoptosis. ${ }^{51}$ An increase in the expression of FLIP/FADD in the testes validated that SiNPs could simultaneously activate FLIP and FADD. However, activation of caspase-8/caspase-3 indicates that FLIP suffered failure in the process of repressing apoptosis. The data demonstrate that SiNPs can induce oxidative stress, which activates RIPK1 pathway and as a result causes apoptosis and necroptosis in the spermatogenic cells of male mice.

On the 75th day after the first dose, SiNPs were almost invisible in the testicular tissue under transmission electron microscopy in the SiNPs group. The sperm concentration, 
B
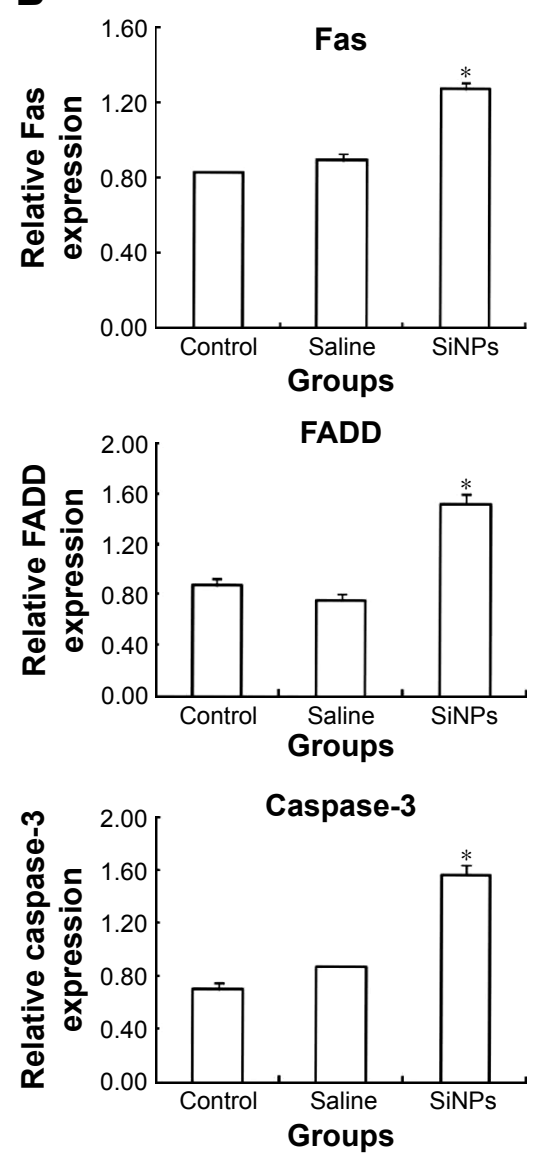

A

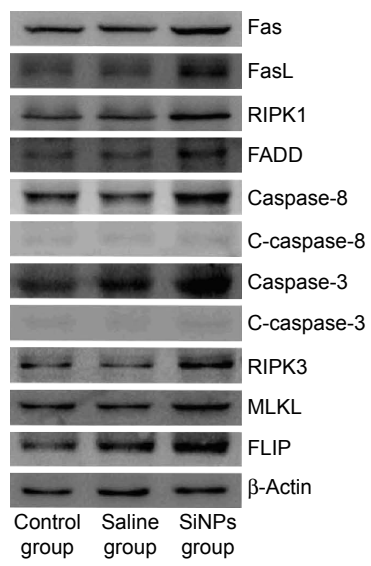

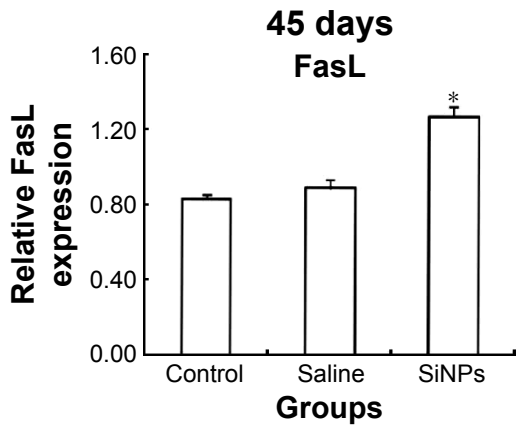

Caspase-8
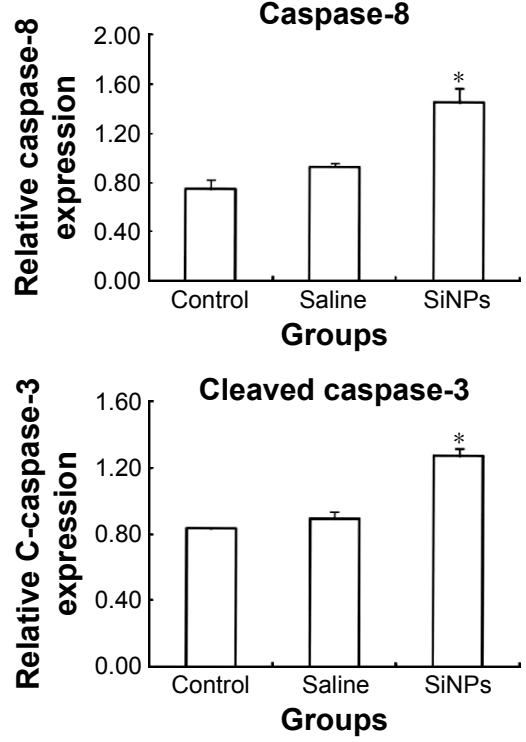

MLKL

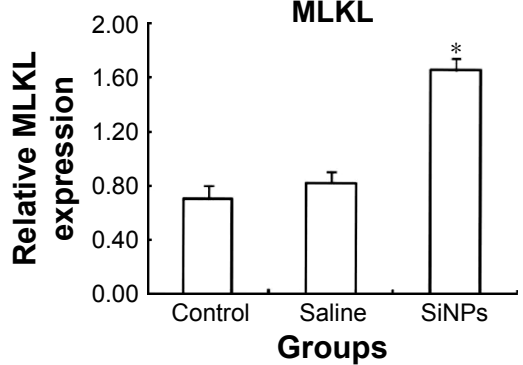

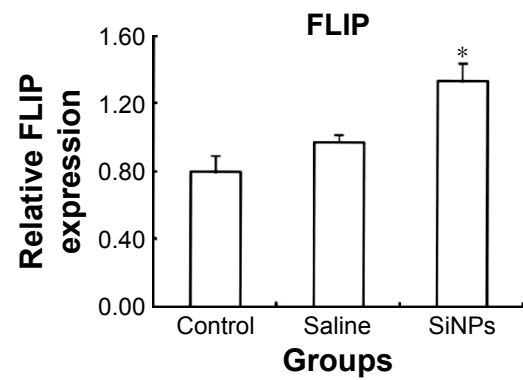

RIPK1
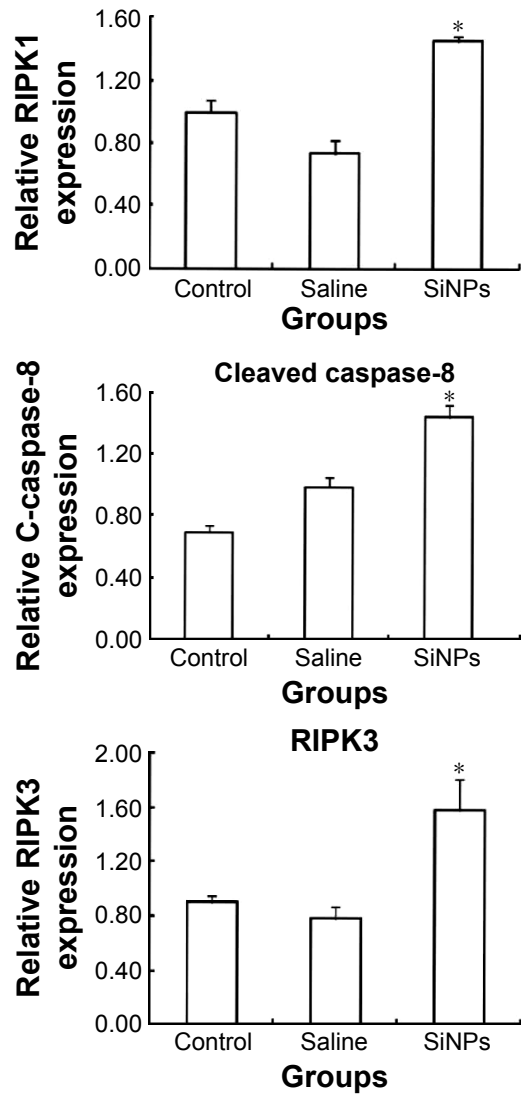

Groups 

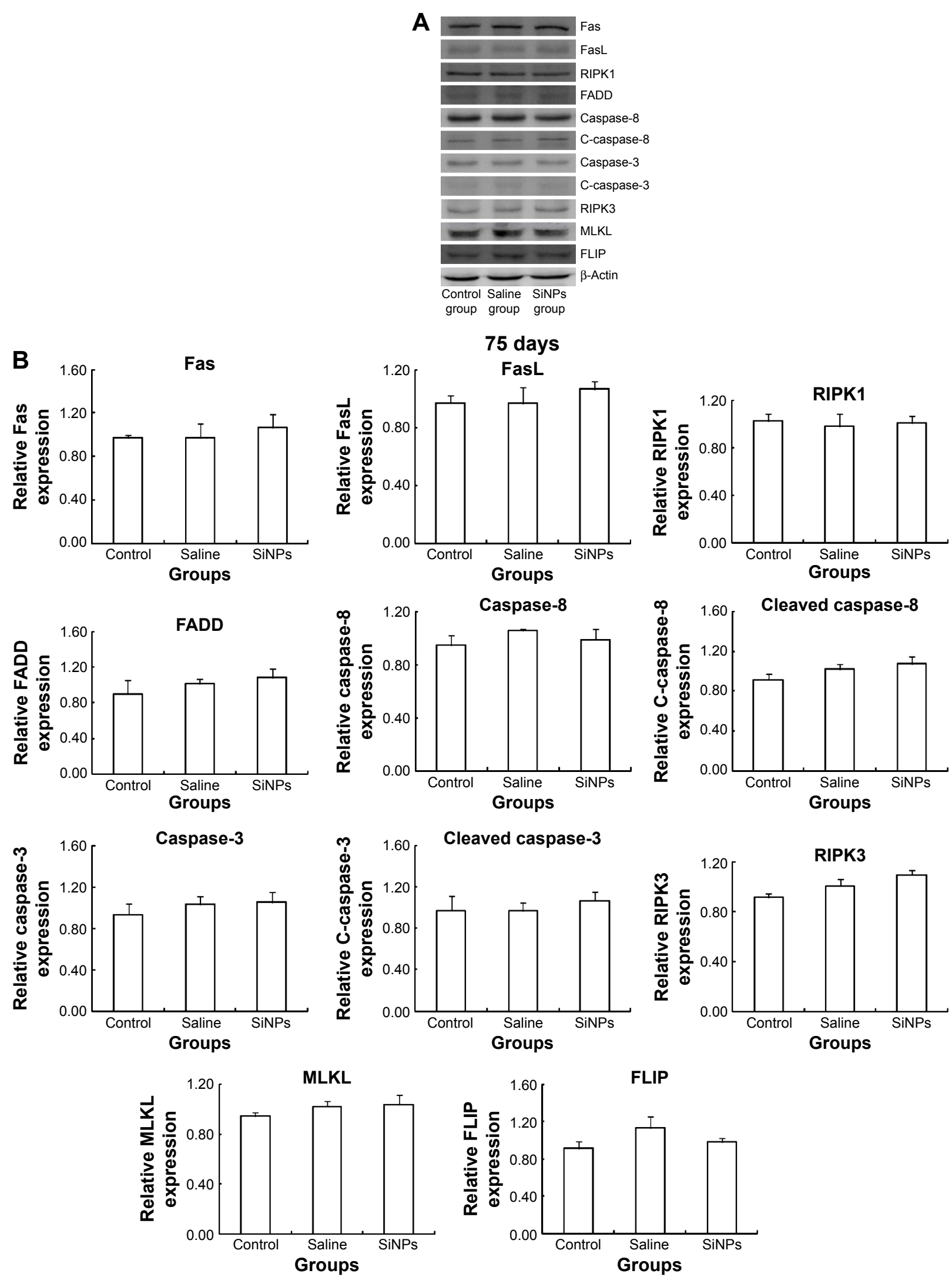

Figure 8 The effects of SiNPs on the expression of proteins in RIPKI signal pathway at 75 days.

Notes: (A) The expression of RIPKI/FADD/caspase-8/cleaved caspase-8/caspase-3/cleaved caspase-3 and RIPK3/MLKL were measured by Western blot assay. (B) Relative densitometric analysis of protein bands were presented. After stopping exposure to SiNPs for 30 days, the effect of SiNPs on the expressions of RIPKI/FADD/caspase-8/ cleaved caspase-8/caspase-3/cleaved caspase-3 and RIPK3/MLKL disappeared. Data are expressed as the mean \pm standard error from three independent experiments. The data indicated that the effects of SiNPs on the expression of protein in the RIPKI signal pathway disappeared after stopping exposure to SiNPs. Abbreviations: SiNPs, silica nanoparticles; FasL, Fas ligand; C-caspase-8, cleaved caspase-8; C-caspase-3, cleaved caspase-3. 


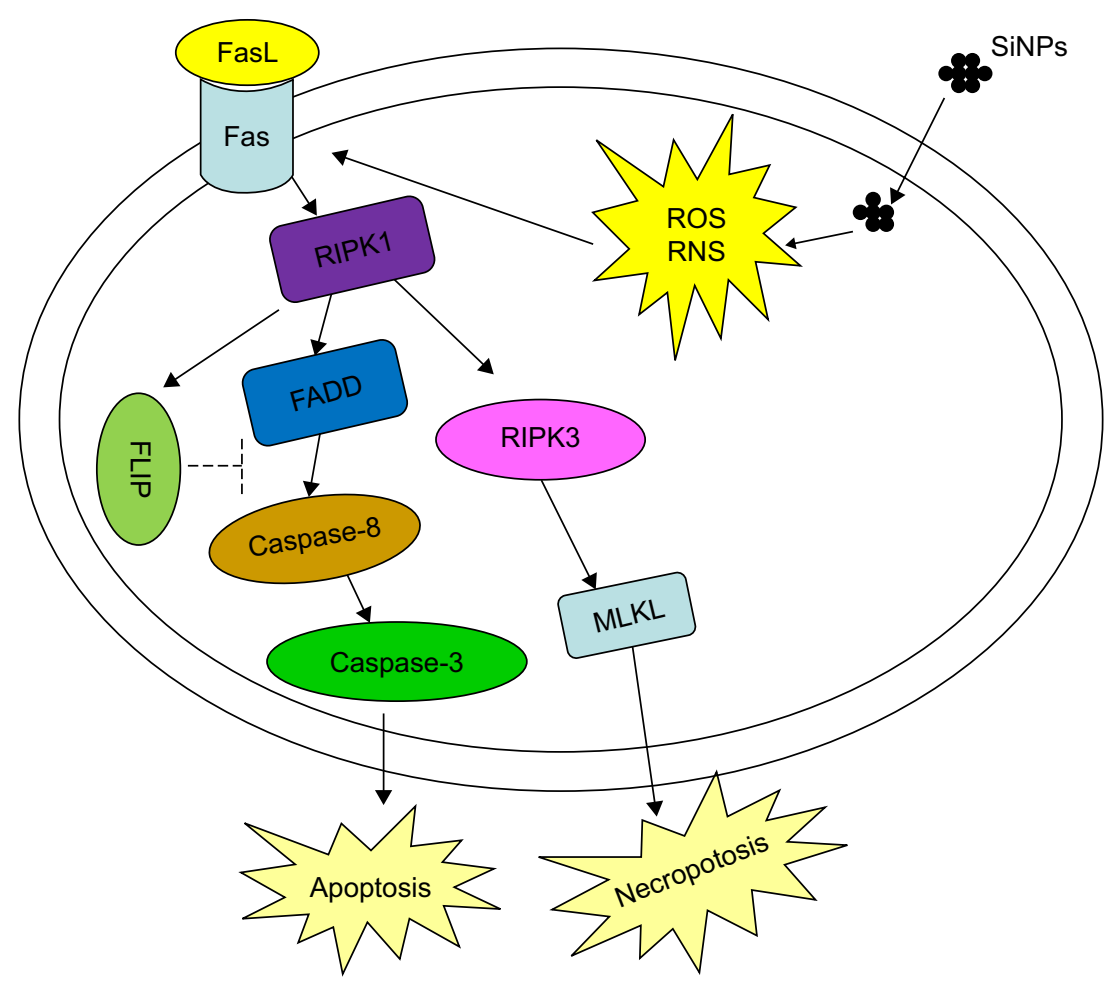

Figure 9 The possible mechanism of SiNPs on spermatogenic cells.

Abbreviations: SiNPs, silica nanoparticles; ROS, reactive oxygen species; RNS, reactive nitrogen species; FasL, Fas ligand.

sperm motility, sperm abnormity, and apoptosis exhibited no significant differences between the SiNPs group and the saline control group. The expression of Fas/FasL/RIPK1/ FADD/caspase-8/caspase-3 and RIPK3/MLKL also showed no significance difference between the SiNPs group and the saline control group. These results suggested that SiNPsinduced damage was conspicuously relieved with the excretion of SiNPs. It was reported that mesoporous hollow SiNPs were removed from the body 4 weeks after stopping exposure to SiNPs, ${ }^{52}$ which resembles our results. Our study indicates that SiNPs-induced damage could be reversible after stopping exposure.

The possible mechanism underlying the SiNPs-induced apoptosis of spermatogenic cells in mice is schematically delineated in Figure 9.

\section{Conclusion}

In this study, SiNPs could decrease the motility and quantity of sperms, cause malformation of sperms, lead to apoptosis and necroptosis in spermatogenic cells, induce oxidative stress in testis, and activate the expression of the proteins Fas/FasL/RIPK1/FADD/caspase-8/caspase-3 and RIPK3/ MLKL on the 45th day after the first dose. In summary, SiNPs could induce apoptosis and necroptosis in spermatogenic cells by activating the RIPK1 pathway, resulting from oxidative stress in male mice, and therefore lead to a decrease in the quality and quantity of sperms. SiNPs-induced damage can recover with the elimination of SiNPs after stopping exposure to SiNPs, which means that the toxicity induced by SiNPs in testes is reversible.

\section{Acknowledgment}

This study was supported by Beijing Natural Science Foundation Program and Scientific Research Key Program of Beijing Municipal Commission of Education (KZ201510025028).

\section{Disclosure}

The authors report no conflicts of interest in this work.

\section{References}

1. Parveen S, Misra R, Sahoo SK. Nanoparticles: a boon to drug delivery, therapeutics, diagnostics and imaging. Nanomedicine. 2012;8(2): $147-166$.

2. Bitar A, Ahmad NM, Fessi H, et al. Silica-based nanoparticles for biomedical applications. Drug Discov Today. 2012;17(19-20):1147-1154.

3. Nabeshi H, Yoshikawa T, Akase T, et al. Effect of amorphous silica nanoparticles on in vitro RANKL-induced osteoclast differentiation in murine macrophages. Nanoscale Res Lett. 2011;6(1):464.

4. Liang X, Chen W, Sun G, et al. Experimental study on new self and mutualaiding occlusive dressing for wound. Chin Med J (Engl). 2014;127(7): $1321-1327$. 
5. Atai M, Pahlavan A, Moin N. Nano-porous thermally sintered nano silica as novel fillers for dental composites. Dent Mater. 2012;28(2): $133-145$

6. Ghio AJ, Kummarapurugu ST, Tong H, et al. Biological effects of desert dust in respiratory epithelial cells and a murine model. Inhal Toxicol. 2014;26(5):299-309.

7. Matassoni L, Pratesi G, Centioli D, et al. Saharan dust contribution to PM10, PM2.5 and PM1 in urban and suburban areas of Rome: a comparison between single-particle SEM-EDS analysis and whole-sample PIXE analysis. J Environ Monit. 2011;13(3):732-742.

8. Theodore L, Kunz RG. Nanotechnology: Environmental Implications and Solutions. John Wiley \& Sons; 2005. DOI:10.1002/ 0471711705 .

9. Baki ME, Miresmaili SM, Pourentezari M, et al. Effects of silver nano-particles on sperm parameters, number of Leydig cells and sex hormones in rats. Iran J Reprod Med. 2014;12(2):139-144.

10. Komatsu T, Tabata M, Kubo-Irie M, et al. The effects of nanoparticles on mouse testis Leydig cells in vitro. Toxicol in Vitro. 2008;22(8): 1825-1831.

11. Yoshida S, Hiyoshi K, Ichinose T, et al. Effect of nanoparticles on the male reproductive system of mice. Int J Androl. 2009;32(4):337-342.

12. Fan YO, Zhang YH, Zhang XP, et al. Comparative study of nanosized and microsized silica on spermatogenesis function of male rats. J Hygiene Res. 2006;35(5):549-553.

13. Lin BC, Xi ZG, Zhang YG, et al. Experimental study on the reproductive damage of male rats induced by micro-nano-scale $\mathrm{SiO}_{2}$. Asian J Ecotoxicol. 2007;2(2):195-201.

14. Xu Y, Wang N, Yu Y, et al. Exposure to silica nanoparticles causes reversible damage of the spermatogenic process in mice. PLOS ONE. 2014;9(7):e101572.

15. Liang S, Xu S, Zhang D, et al. Reproductive toxicity of nanoscale graphene oxide in male mice. Nanotoxicology. 2015;9(1):92-105.

16. Tsuji JS, Maynard AD, Howard PC, et al. Research strategies for safety evaluation of nanomaterials. Part IV: risk assessment of nanoparticles. Toxicol Sci. 2006;89(1):42-50.

17. Kelley AT, Alessi PJ, Fomalik JE, et a1. Investigation and application of nanoparticle dispersions of pigment yellow 185 using organic solvents. ACS Appl Mater Interfaces. 2009;2(1):61-68.

18. Mahmoud KA, Male KB, Hrapovic S, et a1. Cellulose nanocrystal/ gold nanoparticle composite as a matrix for enzyme immobilization. ACS Appl Mater Interfaces. 2009;1(7):1383-1386.

19. Guo C, Xia Y, Niu P, et al. Silica nanoparticles induce oxidative stress, inflammation, and endothelial dysfunction in vitro via activation of the MAPK/Nrf2 pathway and nuclear factor- $\kappa \mathrm{B}$ signaling. Int J Nanomedicine. 2015;10:1463-1477.

20. Melvin A, Vijay R, Chaudhari VR, et al. A facile methodology for the design of functionalized hollow silica spheres. J Colloid Interface Sci. 2010;346(1):265-269.

21. Schneider A, Hampel R, Ibald-Mulli A. et al. Changes in deceleration capacity of heart rate and heart rate variability induced by ambient air pollution in individuals with coronary artery disease. Part Fibre Toxicol. 2010;7:29

22. Mills NL, Tornqvist H, Gonzalez MC, et al. Ischemic and thrombotic effects of dilute diesel-exhaust inhalation in men with coronary heart disease. N Engl J Med. 2007;357(11):1075-1082.

23. Balasubramanian SK, Jittiwat J, Manikandan J, et al. Biodistribution of gold nanoparticles and gene expression changes in the liver and spleen after intravenous administration in rats. Biomaterials. 2010;31(8): 2034-2042.

24. Liang H, Jin C, Tang Y, et al. Cytotoxicity of silica nanoparticles on HaCaT cells. J Appl Toxicol. 2014;34(4):367-372.

25. Zhu J, Liao L, Zhu L, et al. Size-dependent cellular uptake efficiency, mechanism, and cytotoxicity of silica nanoparticles toward HeLa cells. Talanta. 2013;107:408-415

26. Fruijtier-Polloth $\mathrm{C}$. The toxicological mode of action and the safety of synthetic amorphous silica nanostructured material. Toxicology. 2012; 294(2-3):61-79
27. Lu X, Qian J, Zhou H, et al. In vitro cytotoxicity and induction of apoptosis by silica nanoparticles in human HepG2 hepatoma cells. Int J Nanomedicine. 2011;6:1889-1901.

28. Karageorgos N, Patsoukis N, Chroni E, Konstantinou D, Assimakopoulos SF, Georgiou C. Effect of N-acetylcysteine, allopurinol and vitamin $\mathrm{E}$ on jaundice-induced brain oxidative stress in rats. Brain Res. 2006;1111(1):203-212.

29. Gonzalez EJ, Peterson A, Malley S, et al.The effects of tempol on cyclophosphamide-induced oxidative stress in rat micturition reflexes. ScientificWorldJournal. 2015;2015:545048.

30. Duan J, Yu Y, Li Y, et al. Toxic effect of silica nanoparticles on endothelial cells through DNA damage response via Chk1-dependent G2/M checkpoint. PLOS ONE. 2013;8(4):e62087.

31. Singh S, Shi TM, Duffin R, et al. Endocytosis, oxidative stress and IL-8 expression in human lung epithelial cells upon treatment with fine and ultrafine TiO 2: role of the specific surface area and of surface methylation of the particles. Toxicol Appl Pharm. 2007;222(2):141-151.

32. Li R, Zhao X, Chang C, et al. Surface interactions with compartmentalized cellular phosphates explain rare earth oxide nanoparticle hazard and provide opportunities for safer design. ACS Nano. 2014;8(2): 1771-1783.

33. Helle M, Rampazzo E, Monchanin M, et al. Surface chemistry architecture of silica nanoparticles determine the efficiency of in vivo fluorescence lymph node mapping. ACS Nano. 2013;7(10):8645-8657.

34. Shahbazi M, Hamidi M, Mäkilä E, et al. The mechanisms of surface chemistry effects of mesoporous silicon nanoparticles on immunotoxicity and biocompatibility. Biomaterials. 2013;34(31): 7776-7789.

35. Xia T, Kovochich M, Liong M, et al. Cationic polystyrene nanosphere toxicity depends on cell-specific endocytic and mitochondrial injury pathways. ACS Nano. 2008;2(1):85-96.

36. Batandier C, Fontaine E, Keriel C, et al. Determination of mitochondrial reactive oxygen species: methodological aspects. J Cell Mol Med. 2002;6(2):175-187.

37. Sayes CM, Reed KL, Warheit DB. Assessing toxicity of fine and nanoparticles: comparing in vitro measure. Toxicol Sci. 2007;97(1): 163-180.

38. Ryter SW, Kim HP, Hoetzel A. Mechanisms of cell death in oxidative stress. Antioxid Redox Signal. 2007;9(1):49-89.

39. Vanlangenakker N, Vanden Berghe T, Bogaert P, et al. cIAP1 and TAK1 protect cells from TNF-induced necrosis by preventing RIP1/ RIP3-dependent reactive oxygen species production. Cell Death Differ. 2011;18(4):656-665.

40. Zhang DW, Shao J, Lin J, et al. RIP3, an energy metabolism regulator that switches TNF-induced cell death from apoptosis to necrosis. Science. 2009;325(5938):332-336.

41. Yu X, Deng Q, Li W. Neoalbaconol induces cell death through necroptosis by regulating RIPK-dependent autocrine TNF $\alpha$ and ROS production. Oncotarget. 2015;6(4):1995-2008.

42. Xiao X, Chen L, Ouyang Y, et al. Pregnenolone, a cholesterol metabolite, induces glioma cell apoptosis via activating extrinsic and intrinsic apoptotic pathways. Oncol Lett. 2014;8(2):645-650.

43. Wei J, Zhang L, Wang J, et al. Endosulfan inducing blood hypercoagulability and endothelial cells apoptosis via the death receptor pathway in Wistar rats. Toxicol Res. 2015;4:1282-1288.

44. Takahashi N, Vereecke L, Bertrand MJ, et al. RIPK1 ensures intestinal homeostasis by protecting the epithelium against apoptosis. Nature. 2014;513(7516):95-99.

45. Rickard JA, O'Donnell JA, Evans JM, et al. RIPK1 regulates RIPK3MLKL-driven systemic inflammation and emergency hematopoiesis. Cell. 2014;157(5):1175-1188.

46. Dillon CP, Weinlich R, Rodriguez DA, et al. RIPK1 blocks early postnatal lethality mediated by caspase-8 and RIPK3. Cell. 2014;157(5): 1189-1202.

47. Dannappel M, Vlantis K, Kumari S, et al. RIPK1 maintains epithelial homeostasis by inhibiting apoptosis and necroptosis. Nature. 2014; 513(7516):90-94. 
48. Ofengeim D, Yuan J. Regulation of RIP1 kinase signalling at the crossroads of inflammation and cell death. Nat Rev Mol Cell Biol. 2013; 14(11):727-736.

49. Declercq W, Vanden Berghe T, Vandenabeele P. RIP kinases at the crossroads of cell death and survival. Cell. 2009;138(2):229-232.

50. Kataoka T, Tschopp J. N-terminal fragment of c-FLIP(L) processed by caspase- 8 specfically interacts with TRAF2 and induces activation of the NF-кB signaling pathway. Mol Cell Biol. 2004;24(7):2627-2636.
51. Sun J, Luo H, Nie W, et al. Protective effect of RIP and c-FLIP in preventing liver cancer cell apoptosis induced by TRAIL. Int J Clin Exp Pathol. 2015;8(6):6519-6525.

52. Liu T, Li L, Teng X, et al. Single and repeated dose toxicity of mesoporous hollow silica nanoparticles in intravenously exposed mice. Biomaterials. 2011;32(6):1657-1668

\section{Publish your work in this journal}

The International Journal of Nanomedicine is an international, peerreviewed journal focusing on the application of nanotechnology in diagnostics, therapeutics, and drug delivery systems throughout the biomedical field. This journal is indexed on PubMed Central, MedLine, CAS, SciSearch ${ }^{\circledR}$, Current Contents ${ }^{\circledR} /$ Clinical Medicine,
Journal Citation Reports/Science Edition, EMBase, Scopus and the Elsevier Bibliographic databases. The manuscript management system is completely online and includes a very quick and fair peer-review system, which is all easy to use. Visit http://www.dovepress.com/ testimonials.php to read real quotes from published authors. 